\title{
Salivary levels of cortisol and chromogranin A in patients with burning mouth syndrome: A case-control study ${ }^{*}$
}

\author{
Chieko Shigeyama-Haruna ${ }^{1}$, Inho Soh ${ }^{2}$, Akihiro Yoshida ${ }^{2}$, Shuji Awano ${ }^{2}$, Hisashi Anan", \\ Toshihiro Ansai $^{2 \#}$ \\ ${ }^{1}$ Section of Operative Dentistry and Endodontorogy, Department of Odontology, Fukuoka Dental College, Fukuoka, Japan \\ ${ }^{2}$ Division of Community Oral Health Science, Department of Health Promotion, Kyushu Dental College, Kitakyushu, Japan \\ Email: \#ansai@kyu-dent.ac.jp
}

Received 16 January 2013; revised 19 February 2013; accepted 1 March 2013

\begin{abstract}
Burning mouth syndrome (BMS) is a poorly understood oral pain disorder characterized by a painful burning sensation in the oral cavity without any mucosal abnormalities. In this study, we evaluated the salivary cortisol and chromogranin A (CgA) levels of patients with BMS in comparison with age-matched controls. Subjects $(n=114)$ included 81 BMS patients and 33 controls. Patients with BMS were further classified into a subgroup of subjects who occasionally feel a burning sensation (BMS 1), and a subgroup of subjects who always feel a burning sensation (BMS 2). Salivary cortisol and CgA levels were measured using ELISA kits. All individuals with BMS had significantly higher cortisol and CgA levels than the controls did. Furthermore, when comparing the controls with each BMS subgroup, salivary levels of cortisol were significantly higher in both subgroups than controls. In contrast, the level of CgA was significantly higher in the BMS 2 subgroup only. Multiple regression analysis revealed a significant independent association between salivary levels of cortisol and BMS even after adjustment for gender, antidepressant or antianxiety drug use and hypertension (drug-treated). The study revealed that a significant association was observed between salivary cortisol levels and BMS.
\end{abstract}

Keywords: Burning Mouth Syndrome; Cortisol; Chromogranin A

\section{INTRODUCTION}

Burning mouth syndrome (BMS) is a complex disease characterized by burning and/or painful sensations of the mouth, without accompanying abnormal clinical or laboratory findings [1]. Symptoms are usually a burning sensation as well as taste disturbances and/or xerostomia

\footnotetext{
"The authors declare no conflict of interests in the present study.

${ }^{\#}$ Corresponding author.
}

[2]. In Japan, BMS has been reported in approximately $0.8 \%$ of patients who visit dental clinics [3]. Burning mouth complaints are reported more often in women, especially after menopause [4]. The etiology is poorly understood, although the proposed etiological factors have been classified as local (oral candidiasis, parafunctional habits, allergy), systemic (diabetes, deficiency of iron, vitamin $\mathrm{B}_{12}$ and folate), psychogenic, and idiopathic [5]. On the other hand, the association between BMS and psychological stress has been little reported to date.

In the present study, we investigated whether the pathophysiologic status of BMS is associated with changes in endocrinologic hormones related to depression and anxiety, which are included in disorders associated with dysregulation of stress system. In general, the stress system consists of brain elements, of which the main components are corticotropin-releasing hormone ( $\mathrm{CRH})$ and the locus ceruleus-norepinephrine/autonomic systems, as well as their peripheral effectors, the hypothalamic-pituitaryadrenal (HPA) axis and sympathoadrenomedullary (SAM) system [6]. A well-known stress-related hormone is cortisol, while its salivary level reliably reflects HPA activiity and has long been used in human psychobiological studies as a biological marker of stress [7]. Further, chromogranin A (CgA), an acidic glycoprotein that is stored and co-released by exocytosis with catecholamines from the adrenal medulla and sympathetic nerve endings [8], is reported to be released into saliva from salivary glands including the submandibular gland [9]. We recently found significant associations between salivary cortisol and $\mathrm{CgA}$ level and symptoms of oral dryness and reduced salivary flow [10]. The purpose of the present study was to investigate the salivary cortisol and $\mathrm{CgA}$ levels of patients with BMS in comparison with age-matched controls.

\section{SUBJECTS AND METHODS}

\subsection{Subjects}

A case-control study was done in which a group of subjects with BMS was compared with age-matched con- 
trols. A total of 114 subjects, including 81 BMS patients and 33 controls, participated in the study. The BMS patients (male 15 , female 66 , mean age $63.8 \pm 1.5$ years old) were outpatients who treated for BMS at Kyushu Dental College Hospital in Fukuoka Prefecture, Japan. The diagnosis of BMS was established when the patient showed normal oral mucosa, no medical/physical cause for BMS (lichen planus, neuralgia, chronic pain conditions in other regions and geographic tongue), hematologic and laboratory findings within normal limits, presence of the burning sensation for at least 6 months [11]. Patients with BMS were further classified into a subgroup of subjects who occasionally feel a burning sensation (BMS $1, \mathrm{n}=41$ ), and a subgroup of subjects who always feel a burning sensation (BMS 2, $\mathrm{n}=40$ ). The control subjects (male 7, female 26, mean age $67.9 \pm 0.7$ years old) were healthy volunteers $(n=33)$. All subjects understood this study and provided written informed consent. The study protocol was reviewed and approved by the Ethics Committee of Kyushu Dental College (No. 04071007).

Before beginning the Examinations, each subject was asked to respond to a survey consisting of questions related to general medical condition, medication usage, and current smoking status. In addition, self-rated overall health status was determined by their answer to the question, "How do you feel about your recent general health condition?", with the following responses noted: "good", "fair" and "poor". Xerogenic drugs were considered to include antihypertensive agents, antihistamines, analgesics, diuretics, hypnotics, antidepressants, and antianxiety drugs.

\subsection{Biomarker Analyses}

Saliva samples were collected from all subjects between 9:00 $\mathrm{AM}$ and 11:00 $\mathrm{AM}$ to minimize any circadian rhythm effects, after they refrained from oral intake, tooth brushing, and smoking for at least 2 hours prior to collection. Subjects with complete or removable partial dentures kept them in their mouth during saliva collection. Collection of stimulated whole saliva was performed using sterilized tubes. The subjects were first asked to swallow all saliva in the mouth, then chew paraffin for 3 minutes at a constant pace of 60 times per minute, which was monitored with an electric metronome. Collected samples were placed on ice immediately and the salivary flow rate $(\mathrm{mL} / \mathrm{min})$ was estimated by measuring the volume of saliva collected in the tube. Thereafter, the saliva samples were frozen at $-30^{\circ} \mathrm{C}$ until further analysis.

The concentration of cortisol in saliva $(\mathrm{nmol} / \mathrm{L})$ was determined using a salivary cortisol enzyme immunoassay kit (Salimetrics, State College, PA), with a lower sensitivity limit of $0.19 \mathrm{nmol} / \mathrm{L}$, and that of $\mathrm{CgA}$
(pmol/mL) was determined using a YK070 Chromogranin A (Human) electroimmunoassay kit (Yanaihara Institute, Fujinomiya, Japan), with a lower sensitivity limit of $0.01 \mathrm{pmol} / \mathrm{mL}$. Both biomarkers were also measured as absolute amounts, i.e., the amount secreted into the oral cavity per minute, to determine output. To obtain the output value, the mean flow rate and concentration values were multiplied.

\subsection{Statistical Analyses}

Power analysis and sample size estimation were performed using the software G-power, ver. 3.0. The statistical power of this study was found to be $80 \%$ (with sample size: $\mathrm{n} 1: 33, \mathrm{n} 2: 81$ ), an effect size of 0.5 , and $\alpha$ value of 0.05 (2-tailed $t$ test with accuracy mode), which showed reasonable power. In order to assess differences among the groups, a chi-square test was used for categorized variables and a Kruscal-Wallis test or ANOVA for continuous variables. A Scheffe test and Steel-Dwass test of multiple groups were applied after the Kraskal-Wallis test. All statistical software package SPSS (version 11.0 for Windows; SPSS Japan, Tokyo, Japan). The level of statistical significance was set at 0.05 for all of the analyses.

\section{RESULTS}

The demographic characteristics for the 81 BMS patients and 33 controls are presented. There were significant differences between the antidepressant or antianxiety drug use and hypertension (drug-treated), and differences were not significant regarding gender, age, number of teeth, current smoking status, diabetes (drug-treated), and self-rated health status (Table 1).

We compared salivary flow rate (stimulated), and Salivary levels of cortisol and CgA between the BMS patients and controls. Although salivary flow rate was no differences in both, all subjects with BMS had significantly higher cortisol and $\mathrm{CgA}$ levels than the controls regarding to concentration. In output, there was significantly higher cortisol in the BMS patients than controls (Table 2).

We compared between the controls and each BMS subgroup for salivary levels of cortisol and CgA. Salivary levels of cortisol were significantly higher in both BMS subgroups than controls both concentration and output. In contrast, the level of $\mathrm{CgA}$ was significantly higher than in controls in the BMS 2 subgroup only. When the differences between the two subgroups were analyzed, the levels of cortisol were higher in the BMS 2 subgroup; however, no significant difference was observed between the subgroups in regards to the levels of CgA (Table 3).

To determine whether levels of cortisol and $\mathrm{CgA}$ were 
Table 1. Descriptive characteristics of subjects.

\begin{tabular}{|c|c|c|c|}
\hline & Controls & BMS & $P$ value \\
\hline Number of subjects & 33 & 81 & \\
\hline Female & $26(79)$ & $66(81)$ & $\mathrm{NS}^{\dagger}$ \\
\hline Age & $67.9(0.7)$ & $63.8(1.5)$ & $\mathrm{NS}^{*}$ \\
\hline Number of teeth & $21.9(1.2)$ & $23.1(0.8)$ & $\mathrm{NS}^{*}$ \\
\hline Prescribed antidepressants or anti-anxiety drugs & $0(0)$ & $17(21)$ & $0.003^{\dagger}$ \\
\hline Current smoking status & $3(9)$ & $7(9)$ & $\mathrm{NS}^{\dagger}$ \\
\hline Hypertension (drug-treated) & $0(0)$ & $15(19)$ & $0.005^{\dagger}$ \\
\hline Diabetes (drug-treated) & $0(0)$ & $2(2)$ & $\mathrm{NS}^{\dagger}$ \\
\hline Self-rated health status: (good) & $14(42)$ & $19(23)$ & \\
\hline Self-rated health status: (fair) & $16(48)$ & $46(57)$ & $\mathrm{NS}^{\dagger}$ \\
\hline Self-rated health status: (poor) & $3(9)$ & $16(20)$ & \\
\hline
\end{tabular}

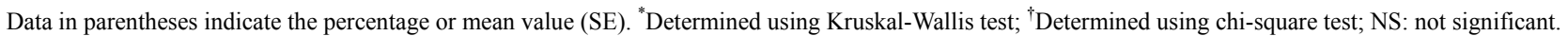

Table 2. Comparison of salivary flow rate and cortisol and $\mathrm{CgA}$ in patients with BMS and controls.

\begin{tabular}{|c|c|c|c|c|}
\hline & & Controls $(\mathrm{N}=33)$ & $\operatorname{BMS}(\mathrm{N}=81)$ & $P$ value \\
\hline \multirow{2}{*}{ Stimulated salivary flow rate $(\mathrm{mL} / \mathrm{min})$} & Median & 1.00 & 0.90 & \multirow{2}{*}{ NS } \\
\hline & 25th, 75 th percentile & $0.70,1.25$ & $0.50,1.55$ & \\
\hline \multicolumn{5}{|l|}{ (A) Concentration } \\
\hline \multirow{2}{*}{ Cortisol (nmol/L) } & Median & 5.23 & 12.41 & \multirow{2}{*}{$<0.001$} \\
\hline & 25th, 75th percentile & $3.27,6.41$ & $4.69,41.38$ & \\
\hline \multirow{2}{*}{$\mathrm{CgA}(\mathrm{pmol} / \mathrm{mL})$} & Median & 3.00 & 4.27 & \multirow{2}{*}{0.030} \\
\hline & 25th, 75th percentile & $1.41,4.56$ & $1.42,7.21$ & \\
\hline \multicolumn{5}{|l|}{ (B) Output } \\
\hline \multirow{2}{*}{ Cortisol (pmol/min) } & Median & 5.63 & 11.17 & \multirow{2}{*}{0.010} \\
\hline & 25th, 75th percentile & $3.23,7.35$ & $2.76,40.00$ & \\
\hline \multirow{2}{*}{$\mathrm{CgA}(\mathrm{pmol} / \mathrm{min})$} & Median & 2.95 & 3.08 & \multirow{2}{*}{ NS } \\
\hline & 25 th, 75 th percentile & $1.02,4.16$ & $1.32,5.55$ & \\
\hline
\end{tabular}

Controls: healthy volunteers as a control; Data are presented as both concentration (A) and total amount secreted (B); CgA: chromogranin A; ${ }^{*}$ Determined using Mann-Whitney U test; NS: not significant.

Table 3. Comparison of salivary flow rate and cortisol and $\mathrm{CgA}$ in patients with BMS and controls.

\begin{tabular}{|c|c|c|c|c|c|}
\hline & & Controls $(\mathrm{N}=33)$ & BMS $1(\mathrm{~N}=41)$ & BMS $2(\mathrm{~N}=40)$ & $P$ value \\
\hline \multirow{2}{*}{ Stimulated salivary flow rate $(\mathrm{mL} / \mathrm{min})$} & Median & 1.00 & 0.90 & 0.90 & \multirow{2}{*}{ NS } \\
\hline & 25th, 75 th percentile & $0.70,1.25$ & $0.60,1.60$ & $0.50,1.38$ & \\
\hline \multicolumn{6}{|l|}{ (A) Concentration } \\
\hline \multirow{2}{*}{ Cortisol (nmol/L) } & Median & 5.23 & $18.84^{\dagger}$ & $34.21 *^{\dagger}$ & \multirow{2}{*}{$<0.001$} \\
\hline & 25 th, 75 th percentile & $3.27,6.41$ & $3.31,20.14$ & $5.10,53.80$ & \\
\hline \multirow{2}{*}{$\mathrm{CgA}(\mathrm{pmol} / \mathrm{mL})$} & Median & 3.00 & 5.02 & $6.25 *^{*^{\dagger}}$ & \multirow{2}{*}{0.031} \\
\hline & 25th, 75 th percentile & $1.41,4.56$ & $1.28,6.82$ & $1.81,8.29$ & \\
\hline \multicolumn{6}{|l|}{ (B) Output } \\
\hline \multirow{2}{*}{ Cortisol (pmol/min) } & Median & 5.63 & 19.32 & $28.54^{*, \dagger}$ & \multirow{2}{*}{0.004} \\
\hline & 25th, 75 th percentile & $3.23,7.35$ & $2.10,24.97$ & $3.41,46.05$ & \\
\hline \multirow{2}{*}{$\mathrm{CgA}(\mathrm{pmol} / \mathrm{min})$} & Median & 2.95 & 3.70 & 4.61 & \multirow{2}{*}{ NS } \\
\hline & 25th, 75 th percentile & $1.02,4.16$ & $1.13,5.03$ & $1.59,6.23$ & \\
\hline
\end{tabular}

Controls: healthy volunteers as a control; BMS 1: subjects who occasionally feel a burning sensation; BMS 2: subjects who always feel a burning sensation; Data are presented as both concentration (A) and total amount secreted (B); CgA: chromogranin A; ${ }^{\S}$ Kruscal-Wallis test; ${ }^{*}$ Versus controls, as determined using Scheffe's test for multiple comparisons $(P<0.05)$; ${ }^{\dagger}$ Versus controls, as determined using Steel-Dwass test for multiple comparisons $(P<0.05)$. NS: not significant. 
associated with the severity and extent of BMS, we performed multiple regression analysis by gender, antidepressant or antianxiety drug use and hypertension (drugtreated). Significant associations were found between cortisol levels and both BMS subgroups with final models. There were no significant associations between BMS subgroups and CgA levels with final models (Table 4).

\section{DISCUSSION}

In the present case-control study, we investigated HPA and SAM responses in outpatients with BMS and found that elevated levels of cortisol were associated with BMS. It has been reported that patients with BMS exhibit significantly more symptoms of depression, anxiety [12, 13], but those assessment of stress were performed through only the questionnaires. On the other hand, the association between BMS and stress-related hormones has been to date overlooked, with only two known human studies of the associations between cortisol in saliva and BMS [14,15]. Amenabar et al. [14] evaluated the association between anxiety and salivary cortisol levels in patients with BMS, and found that BMS group showed higher anxiety and salivary cortisol levels when compare with the control group: the BMS group values were approximately 1.4 times the control group regarding to cortisol. In the present study, we obtained the similar result to theirs that the BMS group values were approximately 2 times the control group regarding to salivary cortisol. In contrast, Lopez-Jornet et al. [15] concluded that no significant association with salivary cortisol between control and BMS patients. However, in both studies, limited number of subjects was analyzed; 30 patients with BMS in the former, and 9 patients in the latter, and then statistical power did not appear to be expected so much. Since the present study has been performed in the larger samples (81 patients and 33 controls), stronger evidence would be obtained. Moreover, we analyzed the difference between BMS subgroups and controls. The level of BMS 1 was approximately 3.6 times compared to the controls, and that of BMS 2 was approximately 6.5 times compared to the control, which showed that patients with severe BMS were higher cortisol levels with a linear trend. In the present study, we also studied the response of $\mathrm{CgA}$, another stress-related hormone, and BMS. Salivary levels of patients with BMS were higher than those of controls in the levels of both hormones, but a weaker association between salivary $\mathrm{CgA}$ and BMS was observed. The $\mathrm{CgA}$ is thought to be released with catecholamines in response to stimulation of the autologous nervous system by psychological stress, but its reactivity with physical stress is known to be low. In contrast, cortisol is highly reactive in the presence of psychological as well as physical stress [16,17]. The discrepancy between cortisol and $\mathrm{CgA}$ levels in the response to BMS may be partly explained by differences in sensitivity to stress.

The measurement of biomarkers in saliva has many advantages, as it is stress-free and non-invasive, and allows for frequent and rapid sampling, whereas diurnal rhythm, artificial changes due to food or drinking substances, and blood-contamination are some of the disadvantages.

The present study has some limitations. First, whether the present biomarkers are useful as predictors for BMS remains unclear, because the design of the present study was cross-sectional. Also, the causes and effects of salivary biomarkers remain unclarified by the present study. Second, levels in saliva should have been investigated at different periods of time due to diurnal hormone rhythm, though BMS group always presented higher cortisol values in the 3 periods of time of the day according to the report by Amenabar et al. [14]. An intervention study is required to validate and extend the associations.

\section{CONCLUSION}

A significant association was observed between salivary cortisol levels and BMS, which suggests an association with the HPA response and symptoms of BMS. Further

Table 4. Multiple regression analysis of the effects of explanatory variables on salivary biomarkers.

\begin{tabular}{|c|c|c|c|c|c|c|c|c|c|c|c|c|}
\hline & \multicolumn{12}{|c|}{ Dependent variables } \\
\hline & \multicolumn{3}{|c|}{ Cortisol (nmol/L) } & \multicolumn{3}{|c|}{ Cortisol (pmol/min) } & \multicolumn{3}{|c|}{$\mathrm{CgA}(\mathrm{pmol} / \mathrm{mL})$} & \multicolumn{3}{|c|}{$\mathrm{CgA}(\mathrm{pmol} / \mathrm{min})$} \\
\hline & $\beta$ & $\mathrm{t}$ & $P$ & $\beta$ & $\mathrm{t}$ & $P$ & $\beta$ & $\mathrm{t}$ & $P$ & $\beta$ & $\mathrm{t}$ & $P$ \\
\hline \multicolumn{13}{|l|}{ Crude } \\
\hline BMS 1 & 0.317 & 2.834 & 0.006 & 0.313 & 2.794 & 0.007 & 0.236 & 2.060 & 0.043 & 0.131 & 1.121 & 0.266 \\
\hline BMS 2 & 0.505 & 4.925 & $<0.001$ & 0.476 & 4.563 & $<0.001$ & 0.340 & 3.043 & 0.003 & 0.247 & 2.145 & 0.035 \\
\hline \multicolumn{13}{|c|}{ Final model ${ }^{*}$} \\
\hline BMS 1 & 0.261 & 2.231 & 0.029 & 0.219 & 1.740 & 0.086 & 0.205 & 1.863 & 0.067 & 0.137 & 1.217 & 0.228 \\
\hline BMS 2 & 0.416 & 4.132 & $<0.001$ & 0.387 & 3.825 & $<0.001$ & 0.188 & 1.609 & 0.112 & 0.156 & 1.425 & 0.159 \\
\hline
\end{tabular}

BMS 1: subjects who occasionally feel a burning sensation; BMS 2: subjects who always feel a burning sensation; ${ }^{*}$ Adjusted for gender, antidepressant or antianxiety drug use and hypertension (drug-treated); $\mathrm{CgA}$ : chromogranin $\mathrm{A} ; \beta$ : regression coefficient; The reference group is the controls. 
studies of these salivary biomarkers may lead to the development of a method monitoring the levels of anxiety and depression in subjects suffering from BMS in the future.

\section{ACKNOWLEDGEMENTS}

This work was partly supported by the Grants-in-Aid for Scientific Research 23593096 (IS), 22390403 (TA), and 21791871 (CS).

\section{REFERENCES}

[1] Miriam, G. Joel, B.E. and Meir, G. (2002) Burning mouth syndrome. American Family Physician, 65, 615-620.

[2] Meir, G., Sol Jr., S. and Henry, C. (1991) Clinical characteristics and management outcome in the burning mouth syndrome. An open study of 130 patients. Oral Surgery Oral Medicine Oral Pathology Oral Radiology and Endodontology, 72, 192-195.

[3] Kitajima, S., Koga, C., Sakaino, H., Kameyama, T. and Takamuki, K. (2001) Changes in the character istics of dental psychosomatic patients visiting the department of oral surgery at a university hospital in Japan: A comparison of the clinical statistics collected in the course of treatment in 1989 and 1998. Japanese Journal of Psychosomatic Dentistry, 16, 43-49.

[4] Michele, D.M., Stefano, F., Lucio, L.R. and Stefania, L. (2005) The diagnosis of burning mouth syndrome represents a challenge for clinicians. Journal of Orofacial Pain, 19, 168-173.

[5] Scala, A., Checchi, L., Montevecch, M. and Marini, I. (2003) Update on burning mouth syndrome: Over view and patient management. Critical Reviews in Oral Biology and Medicine, 14, 275-291. doi:10.1177/154411130301400405

[6] George, P.C. and Philip, W.G. (1992) The consepts of stress and stress system disorders. Overview of physical and behavioral homeostasis. The Journal of the American Medical Association, 267, 1244-1252. doi:10.1001/jama.1992.03480090092034

[7] Sally, S.D. and Margaret, E.K. (2004) Acute stressors and cortisol responses: A theoretical integration and synthesis of laboratory research. Psychopathologic Bulletin, 130, 355-391. doi:10.1037/0033-2909.130.3.355

[8] Daniel, T.O. and Keevin, N.B. (1984) Radioimmunoassay of chromogranin A in plasma as a measure of exocytotic sympathoadrenal activity in normal subjects and pa- tients with pheochromocytoma. New England Journal of Medicine, 311, 764-770.

doi:10.1056/NEJM198409203111204

[9] Saruta, J., Tukinoki, K., Sasaguri, K., Ishii, H., Yasuda, M., Osamura, Y., et al. (2005) Expression and localization of chromogranin A gene and protein in human submandibular gland. Cells Tissues Organs, 180, 237-244. doi:10.1159/000088939

[10] Shigeyama, C., Ansai, T., Awano, S., Soh, I., Yoshida, A., Hamasaki, T., et al. (2008) Salivary levels of cortisol and chromogranin A in patients with dry mouth compared with age-matched controls. Oral Surgery Oral Medicine Oral Pathology Oral Radiology and Endodontology, 106, 833-839. doi:10.1016/j.tripleo.2008.07.005

[11] Philip-John, L., Ruth, F., Sally-Anne, E., Caroline, P. and Terry, R. (2005) Vulnerability and presenting symptoms in burning mouth syndrome. Oral Surgery Oral Medicine Oral Pathology Oral Radiology and Endodontology, 99, 48-54. doi:10.1016/j.tripleo.2004.01.021

[12] Rojo, L., Silvestre, F.J., Bagan, J.V. and De Vicente, T. (1994) Prevalence of psychopathology in burning mouth syndrome. A comparative study among patients with and without psychiatric disorders and controls. Oral Surgery Oral Medicine Oral Pathology, 78, 312-316. doi:10.1016/0030-4220(94)90060-4

[13] Carlson, C.R., Miller, C.S. and Reid, K.I. (2000) Psychosocial profiles of patients with burning mouth syndrome. Journal of Orofacial Pain, 14, 59-64.

[14] Jose, M.A., Josiane, P., Juliana, B.H., Fernando, N.H., Denise, B., Francisco, L., et al. (2008) Anxiety and salivary cortisol levels in patients with burning mouth syndrome: Case-control study. Oral Surgery Oral Medicine Oral Pathology Oral Radiology and Endodontology, 105 460-465. doi:10.1016/j.tripleo.2007.10.002

[15] Lopez-Jornet, P., Camacho-Alonso, F. and AndujarMateos, M.P. (2009) Salivary cortisol, stress, and quality of life in patients with burning mouth syndrome. Journal of European Dermatology Venereology, 23, 1197-1223.

[16] Toda, M., Den, R., Nagasawa, S., Kitamura, K. and Morimoto, K. (2005) Relationship between lifestyle scores and salivary stress markers cortisol and chromogranin A. Archives of Environmental and Occupational Health, 60 266-269. doi:10.3200/AEOH.60.5.266-269

[17] Nakane, H., Asami, O., Yamada, Y., Harada, T., Matsui, N., Kanno, T., et al. (1998) Salivary chromogranin A as an index of psychosomatic stress response. Biomedical Research, 19, 401-406. 